\title{
Alternating Sign Matrices, Related (0,1)-Matrices, and the Smith Normal Form
}

\author{
Richard A. Brualdi* \\ Geir Dahl \\ March 11, 2018 \\ Dedicated with admiration to Vladimir Sergeichuk \\ on the occasion of his 70th birthday.

\begin{abstract}
We investigate the Smith Normal Form (SNF) of alternating sign matrices (ASMs) and related matrices of 0's and 1's ( $(0,1)$-matrices). We identify certain classes of ASMs and (0,1)-matrices whose SNFs are (0,1)-matrices. We relate some of our work to various ranks, in particular, the $(0,1)$-rank of a $(0,1)$-matrix, that is, the bipartite partition number of a bipartite graph.
\end{abstract}

Key words. Alternating sign matrix, convex $(0,1)$-matrix, rank, bipartite graph, Smith normal form.

AMS subject classifications. 05B20, 15B48.

\section{Introduction}

An alternating sign matrix, abbreviated to ASM, is an $n \times n(0, \pm 1)$-matrix such that, ignoring 0 's, the 1's and -1 's in each row and column alternate beginning

*Department of Mathematics, University of Wisconsin, Madison, WI 53706, USA. brualdi@math.wisc.edu

${ }^{\dagger}$ Department of Mathematics, University of Oslo, Norway. geird@math.uio.no. Corresponding author. 
and ending with a 1. ASMs can be regarded as generalizations of permutation matrices, since permutation matrices are the ASMs without any -1 's. Some ASMs $A$ containing -1 's are signature equivalent to $(0,1)$-matrices, that is, there may exist diagonal matrices $D_{1}$ and $D_{2}$ all of whose diagonal entries are 1 or -1 , such that $D_{1} A D_{2}$ is a $(0,1)$-matrix. The matrix $D_{1} A D_{2}$ results from $A$ by multiplying certain rows and columns by -1 . The following example illustrates these observations.

\section{Example 1.1.}

\begin{tabular}{|c|c|c|c|c|c|c|c|c|c|}
\hline & 1 & -1 & 1 & -1 & & & & & \\
\hline-1 & & 1 & & & & & 1 & & \\
\hline 1 & 1 & 1 & 1 & & $\rightarrow$ & 1 & 1 & 1 & \\
\hline & 1 & & 1 & & & & 1 & 1 & 1 \\
\hline-1 & & 1 & -1 & 1 & & & & 1 & \\
\hline 1 & & & 1 & & & & & & \\
\hline
\end{tabular}

Recall the three basic unimodular row operations on an integral matrix: (i) interchanging two rows, (ii) multiplying a row by -1 , and (iii) adding an integral multiple of one row to another row. Operations (i), (ii) and (iii) are called elementary row operations, abbreviated to EROs. Unimodular column operations are defined similarly and we get ECOs. For simplicity, we refer to both of these operations as elementary operations or EOs. Thus $D_{1} A D_{2}$ results from $A$ by a sequence of EOs of type (ii).

Let $A$ be an $m \times n$ integral matrix. Then, by EOs, $A$ can be brought to diagonal form

$$
\left[\begin{array}{cccc|c}
e_{1} & 0 & \cdots & 0 & \\
0 & e_{2} & \cdots & 0 & O_{r, n-r} \\
\vdots & \vdots & \ddots & \vdots & \\
0 & 0 & \cdots & e_{r} & \\
\hline & & & & \\
& & O_{m-r, r} & & O_{m-r, n-r}
\end{array}\right]
$$

where $r$ is the rank of $A$, and $e_{1}, e_{2}, \ldots, e_{r}$ are positive integers satisfying the divisibility conditions $e_{1}\left|e_{2}, e_{2}\right| e_{3}, \ldots, e_{r-1} \mid e_{r}$. The matrix (1) is called the Smith normal form of $A$, denoted as $\operatorname{SNF}(A)$, and $e_{1}, e_{2}, \ldots, e_{r}$ are the invariant factors of $A$. The invariant factors of $A$ are unique and, in fact, $e_{i}=d_{i} / d_{i-1}$ where $d_{i}$ is the greatest common divisor of all determinantal minors of size $i(1 \leq i \leq r)$ and $d_{0}=1$. The positive integers $d_{1}, d_{2}, \ldots, d_{r}$ are the elementary divisors of $A$. If $A$ is nonsingular, then $r=n$ and $e_{1} e_{2} \cdots e_{n}=|\operatorname{det}(A)|$. Note that the SNF of a matrix is, in particular, invariant under permutations of its rows and of its columns. The paper [18] surveys some aspects of the Smith normal form of combinatorially defined matrices. 
We say that $A$ has a $(0,1)-S N F$ provided that its invariant factors all equal 1 , that is, provided

$$
\operatorname{SNF}(A)=\left[\begin{array}{ll}
I_{r} & O \\
O & O
\end{array}\right]
$$

where $r$ is the rank of $A$. Neither the Smith normal form of an ASM nor of a $(0,1)$-matrix needs to be a $(0,1)$-matrix as shown in the following examples.

Example 1.2. We have

$$
\operatorname{SNF}\left(\left[\begin{array}{lll}
1 & 1 & 0 \\
0 & 1 & 1 \\
1 & 0 & 1
\end{array}\right]\right)=\left[\begin{array}{lll}
1 & 0 & 0 \\
0 & 1 & 0 \\
0 & 0 & 2
\end{array}\right] .
$$

This follows by observing that this matrix has determinant equal to 2 and all $2 \times 2$ minors have value \pm 1 .

Now consider the following $12 \times 12 \mathrm{ASM} A$ where we use + to denote a 1 and - to denote a -1 :

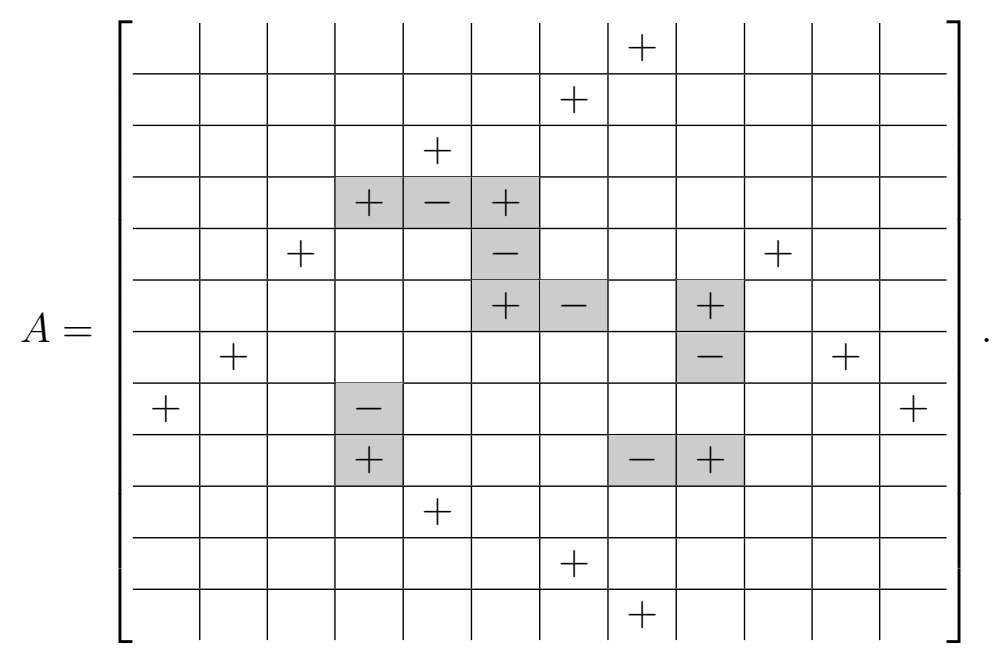

The shaded entries of $A$ indicate a cycle configuration of consecutive entries of the form $(+-+)$ such that any two have at most one entry in common. To find the SNF of $A$ we use each of the 1's in the first three rows and eliminate the nonzeros below, and then we use each of the 1's in the first three columns and eliminate the 
nonzeros to the right. The resulting matrix is

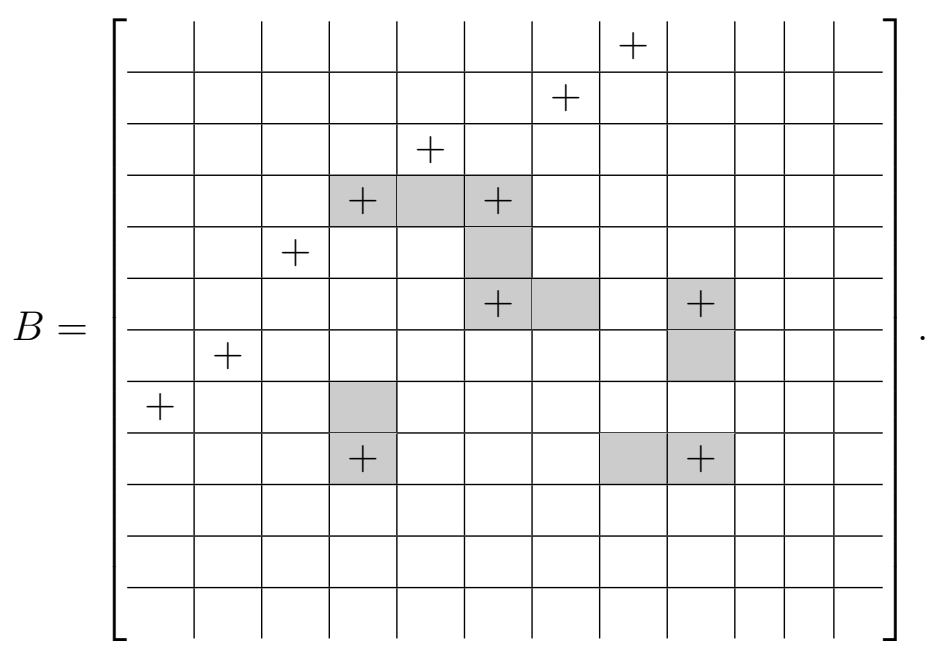

Note the 6 ones in shaded cells now form a bipartite cycle. Permuting rows and columns so that this cycle corresponds to rows and columns $7,8,9$ we get

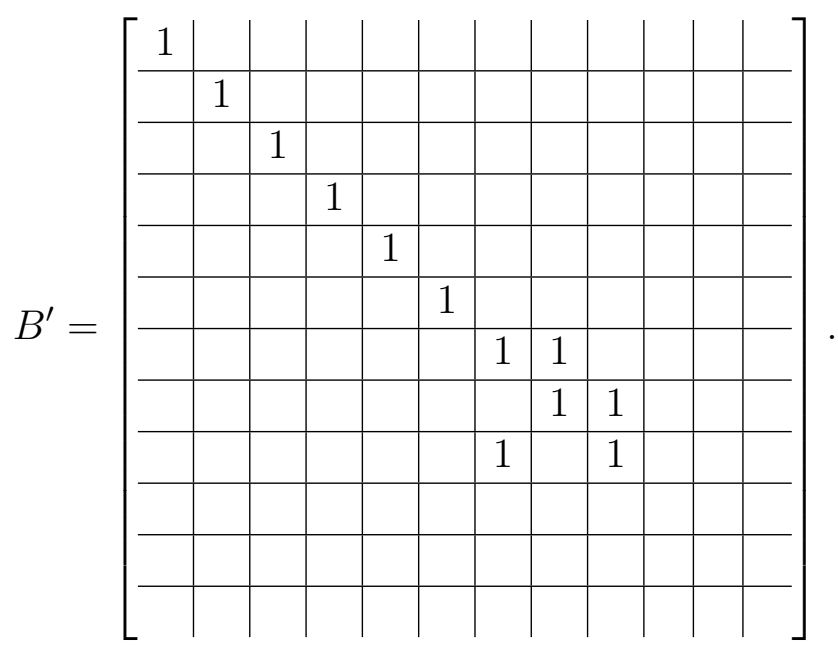

Then (see (3)) $\operatorname{SNF}\left(B^{\prime}\right)=\operatorname{SNF}(A)$ is the diagonal matrix with diagonal

$$
(1,1,1,1,1,1,1,1,2,0,0,0),
$$

and hence $A$ does not have a $(0,1)$-SNF.

If an $n \times n$ integral matrix $A$ satisfies that, for $k=1,2, \ldots, n, A$ contains a minor which is \pm 1 , then it follows that every elementary divisor, and hence every invariant factor, is 1 , so that $A$ has a $(0,1)$-SNF. ASMs satisfying this sufficient condition may have some interesting properties. 
Our goal in this paper is to initiate an investigation of the Smith Normal Form of ASMs and related $(0,1)$-matrices. Note that a $(0, \pm 1)$-matrix $A$ is signature equivalent to a $(0,1)$-matrix if and only if there exist permutation matrices $P$ and $Q$ such that

$$
P A Q=\left[\begin{array}{rr}
A_{11} & -A_{12} \\
-A_{21} & A_{22}
\end{array}\right]
$$

where $A_{11}, A_{12}, A_{21}, A_{22}$ are all $(0,1)$-matrices. The matrix $A_{22}$, and hence $A_{12}$ and $A_{21}$, may be empty. With a $(0, \pm 1)$-matrix $A$ we can associate a bipartite graph with vertex bipartition $U$ (corresponding to the rows) and $W$ (corresponding to the columns), and edges signed as +1 or -1 , to get a signed bipartite graph $G^{ \pm}(A)$. The matrix $A$ is then the signed adjacency matrix of $G^{ \pm}(A)$. A cycle of $G^{ \pm}(A)$ is a positive cycle provided the product of the signs of its edges is +1 , that is, has an even number of edges with sign -1 , and is a negative cycle otherwise. It follows easily that if $A$ is signature equivalent to a $(0,1)$-matrix (see (4)), then all cycles of $G^{ \pm}(A)$ are positive. The following known theorem $[9,13]$ asserts that the converse holds as well.

Theorem 1.3. Let $A$ be an $m \times n(0, \pm 1)$-matrix. Then $A$ is signature equivalent to a $(0,1)$-matrix if and only if all cycles of $G^{ \pm}(A)$ are positive.

Proof. For the convenience of the reader we outline a brief proof of the converse. So suppose that all cycles of $G^{ \pm}(A)$ are positive, that is, contain an even number of minus edges. Let $G^{+}$be the bipartite graph obtained from $G^{ \pm}$by deleting its minus edges. Then there does not exist a negative edge of $G$ joining two vertices in the same connected component of $G^{+}$, since such an edge would give a cycle of $G$ with exactly one minus edge. Hence all the minus edges of $G$, if any, join two vertices in different components of $G^{+}$. Let $H$ be the graph whose vertices correspond to the connected components of $G^{+}$with an edge between two connected components if there is a negative edge between them in $G^{ \pm}$. Then $H$ is a bipartite graph for, otherwise, $H$ contains a cycle of odd length and then $G^{ \pm}$contains a negative cycle. The bipartition of the vertices of $H$ induces the partition as given in (4).

\section{Convex (0,1)-Matrices and ASM's}

Let $A=\left[a_{i j}\right]$ be a nonzero $(0, \pm 1)$-matrix. We say that $A$ is row-convex if there does not exist a zero between two nonzeros in any row. The notion of column-convex is defined similarly. If $A$ is both row- and column-convex, we say that $A$ is convex. A $(0,1)$-matrix which is either row-convex or column-convex is usually called an interval matrix. Row-convexity or column-convexity of a $(0,1)$-matrix implies a $(0,1)-\mathrm{SNF}$. 
Theorem 2.1. Let $A$ be an $m \times n(0,1)$-matrix which is row-convex or columnconvex. Then $A$ has a $(0,1)-S N F$.

Proof. Assume that $A$ is column-convex. Then $A$ is an interval matrix, and this implies that $A$ is a totally unimodular matrix [14]. Thus each determinantal minor of $A$ is 0,1 or -1 and hence the invariant factors of $A$ all equal 1 . Hence $A$ has a $(0,1)$-SNF. A similar argument holds when $A$ is row-convex.

If $A$ is not convex, then $A$ may not have a $(0,1)-\mathrm{SNF}$, as the example (3) shows. We note that this connection to totally unimodular matrices was also discussed in [6].

We now consider convex ASM's (sometimes also called dense ASM's). Some special $n \times n$ convex ASM's are the matrices $F_{n}^{k}$, where $1 \leq k \leq n$, defined as: There are +1 's in the positions in the stripes running from position $(1, k)$ to position $(k, 1)$, from $(k, 1)$ to position $(n, n-k+1)$, from $(n, n-k+1)$ to position $(n+1-k, n)$, and from $(n+1-k, n)$ back to position $(1, k)$. Each of the entries within the region bordered by these four stripes is nonzero and so is uniquely determined, and all positions outside of this region are zero. In particular, $F_{n}^{1}$ is the $n \times n$ identity matrix $I_{n}$ and $F_{n}^{n}$ is the permutation matrix $L_{n}$ with 1 's on the back diagonal (running from position $(1, n)$ to $(n, 1)$ ). For example,

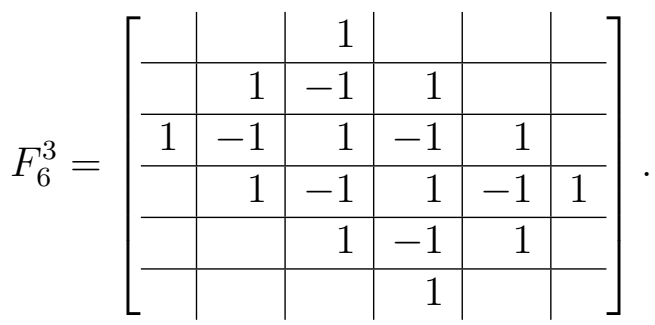

Corollary 2.2. If $A$ is a convex $A S M$, then $A$ has a $(0,1)-S N F$. In particular, the $A S M s F_{n}^{k}$ has a $(0,1)-S N F$.

Proof. Up to permutations of rows and of columns, any convex ASM may be constructed from smaller $F_{n}^{k}$-matrices by putting these as block matrices in a permutation pattern. Therefore, after row and column permutations, $A$ is a direct sum of $F_{n}^{k}$-matrices; thus it is enough to prove the result for matrices $F_{n}^{k}$. Let $S$ be the diagonal matrix with diagonal matrix $\operatorname{diag}(1,-1,1,-1, \ldots)$. Then, see Lemma 2.1 in [7] (and it is easy to check), $S F_{n}^{k} S$ is an all ones matrix or its negative. After possibly multiplying each row by -1 , we obtain the matrix $P_{n}^{k}$ which is obtained from $F_{n}^{k}$ by replacing each -1 by 1 . This $(0,1)$-matrix is convex and thus, by Theorem 2.1 , it has a $(0,1)$-SNF. Since $F_{n}^{k}$ is signature equivalent to $P_{n}^{k}, F_{n}^{k}$ has a $(0,1)$-SNF. The corollary now follows. 
The rank of each ASM $F_{n}^{k}$ was determined in [7].

Theorem 2.1 was proved by using the total unimodularity of $(0,1)$-matrices which are row-convex or column-convex. The conclusion of this theorem can be strengthened with an algorithm for determining the SNF providing also a connection to other related ideas which we now discuss. A characterization of the rank $\rho(A)$ of a real nonzero matrix $A$ can be given in terms of rank 1 matrices: $\rho(A)$ is the smallest integer $r$ such that $A$ is the sum of $r$ rank 1 matrices. If $A$ is a nonnegative matrix, then the nonnegative rank $\rho_{\geq 0}(A)$ of $A$ is the smallest integer $k$ such that $A$ is a sum of $k$ nonnegative matrices of rank 1 . If $A$ is also a $(0,1)$-matrix, then the $(0,1)$-rank $\rho_{0,1}(A)$ is the smallest integer $t$ such that $A$ is the sum of $t$ rank $1(0,1)$-matrices. We clearly have

$$
\rho(A) \leq \rho_{\geq 0}(A) \leq \rho_{0,1}(A) \text { for a }(0,1) \text {-matrix } A
$$

and these inequalities may be strict [19]. Both the computations of nonnegative rank and $(0,1)$-rank are known to be NP-hard $[11,12,15,16]$. (To indicate the subtle nature of these kinds of restricted ranks, we remark that Shitov [16] has constructed an example of a $21 \times 21$ nonnegative integral matrix with nonnegative rank equal to 19 which cannot be written as a sum of 19 nonnegative rational matrices of rank 1. See also [4].)

The $(0,1)$-rank of an $m \times n(0,1)$-matrix $A$ can also be formulated in terms of the bipartite graph $G(A)$ with biadjacency matrix equal to $A$. A rank $1(0,1)$-matrix corresponds to a biclique of $A$ and the $(0,1)$-rank of $A$ equals the bipartition number of $G(A)$, that is, smallest number $t$ of bicliques into which the edges of $G(A)$ can be partitioned.

The following algorithm and theorem is formulated for row-convex $(0,1)$-matrices, but holds equally well for column convex $(0,1)$-matrices.

\section{Algorithm for SNF and (0,1)-Rank of a Row-Convex (0,1)-Matrix}

Let $A=\left[a_{i j}\right]$ be an $m \times n$ row-convex $(0,1)$-matrix. Initially, let $j^{*}=0$. The output of the algorithm is a matrix $Q$.

(i) If $j^{*}=n$ or each column $k>j^{*}$ is zero, stop, and let $Q=A$. Otherwise, let $k>j^{*}$ be the smallest index of a nonzero column of $A$ (after column $j^{*}$ ), and let $C_{k}=\left\{i: a_{i k}=1\right\}$ with $c_{k}=\left|C_{k}\right|$.

(ii) Choose $t \in C_{k}$ such that row $t$ of $A$ has the smallest number $p_{t}$ of 1 's in its row, thus determining a $c_{k} \times p_{t}$ submatrix $A^{k, t}$ of all 1 's.

(iii) Subtract row $t$ from each row in $C_{k}$. 
(iv) Subtract column $k$ from each column $s>k$ for which $a_{t s}=1$. (At this point, all the 1's of submatrix $A^{k, t}$ have been replaced with 0's except for $a_{t k}=1$ which is the only 1 in row $t$ and the only 1 in column $k$; we call this 1 a special 1 of $A$.)

(v) Let $j^{*}=k$, and go back to step (i).

Theorem 2.3. Let $A=\left[a_{i j}\right]$ be an $m \times n$ row-convex $(0,1)$-matrix. Then $\rho(A)=$ $\rho_{0,1}(A)$. Using EOs the above algorithm produces a subpermutation matrix $Q$ where all intermediary matrices are also row-convex $(0,1)$-matrices, and the support of each matrix is contained in the support of the previous one. Then by permutations of the rows and of the columns of $Q$ we can obtain the Smith normal form of $A$.

Proof. Since $A$ is row-convex, the matrix $A^{\prime}$ obtained after step (iii) and (iv) using EOs is also row-convex and has rank and $(0,1)$-rank one less than that of $A$. This holds in every iteration, and the submatrix consisting of the $j^{*}$ first columns is a subpermutation matrix. The special 1's produced by the algorithm determine a subpermutation matrix $Q$ whose rank and $(0,1)$-rank are equal and equal those of the original $A$. The other assertions in the theorem follow immediately from the algorithm.

The next example illustrates this algorithm.

Example 2.4. Below we give a row-convex $(0,1)$-matrix and show the reduction to a subpermutation matrix. The special 1's are those in positions $(3,1),(2,2),(1,4)$ and $(4,5)$ and are in boldface.
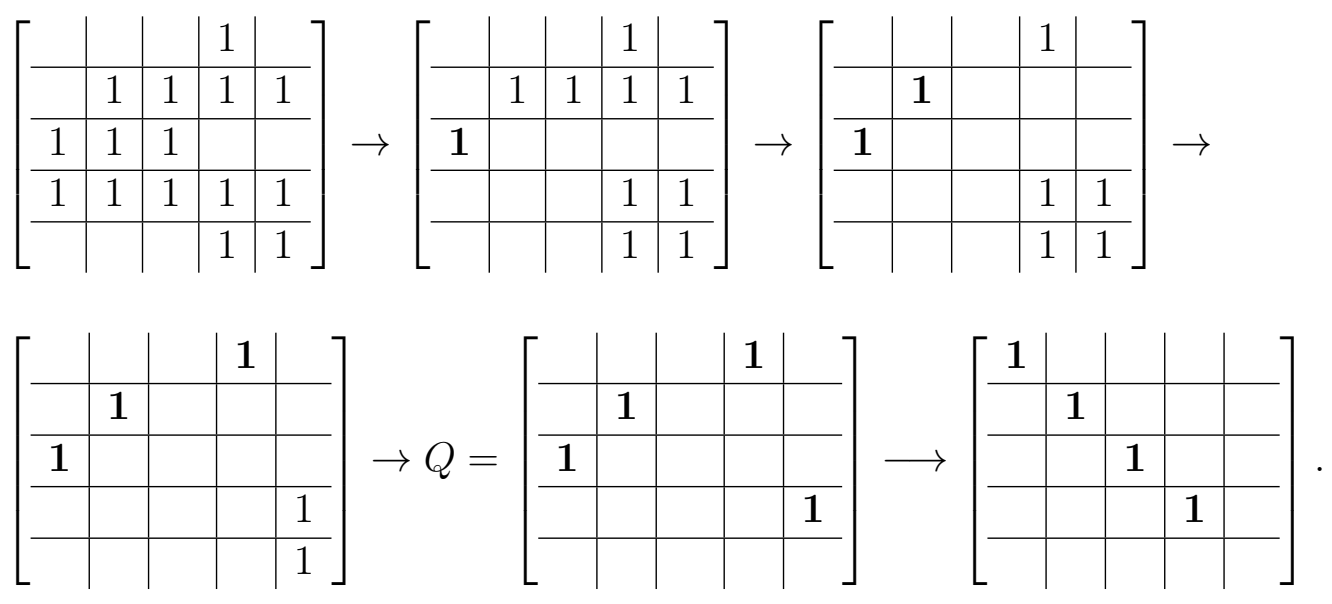
The final example in this section shows that it may not be possible to reduce a convex $(0,1)$-matrix into a subpermutation matrix in such a way that all intermediary matrices are convex $(0,1)$-matrices. Of course, the algorithm above may be applied, but it only assures row-convexity, or column-convexity, but not both properties.

Example 2.5. Let $A$ be the convex $(0,1)$-matrix

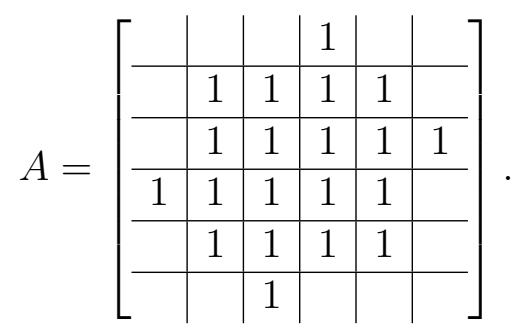

Then it is easy to see that it is impossible to perform a single EO of types (i) or (iii) that results in a new convex $(0,1)$-matrix. For instance, subtracting the first column from one of the next four columns, the resulting affected column will not be convex.

\section{Staircase $(0,1)$-Matrices}

In this section we consider $m \times n$ convex $(0,1)$-matrices whose 1 's form a staircase pattern. Consider integer sequences $1 \leq c_{1} \leq c_{2} \leq \cdots \leq c_{m} \leq n$ and $1 \leq d_{1} \leq d_{2} \leq$ $\cdots \leq d_{m} \leq n$ such that $c_{i} \leq d_{i}$ for $i=1,2, \ldots, m$. The $m \times n(0,1)$-matrix $A=\left[a_{i j}\right]$ such that $a_{i j}=1$ if and only if $c_{i} \leq j \leq d_{i}$ for $i=1,2, \ldots, m$ is a staircase $(0,1)$ matrix; these matrices are both row-convex and column-convex. Staircase matrices are used in [2] in investigating the Bruhat order on the set of $n \times n$ permutation matrices. They also arise in [17] in the investigation of bipartite permutation graphs which we now discuss. In [3], staircase matrices are called saw-toothed chessboards.

Let $\sigma=\left(i_{1}, i_{2}, \ldots, i_{n}\right)$ and $\tau=\left(j_{1}, j_{2}, \ldots, j_{n}\right)$ be permutations of $\{1,2, \ldots, n\}$. The permutation graph $G_{\sigma, \tau}$ determined by $\sigma$ and $\tau$ is the graph with vertex set $\{1,2, \ldots, n\}$ such that there is an edge joining vertices $k$ and $l$ if and only if $k$ precedes $l$ in one of $\sigma$ and $\tau$, and $l$ precedes $k$ in the other. As discussed in [17], the edges of $G_{\sigma, \tau}$ are determined by the permutation diagram $D_{\sigma, \tau}$ which is a $2 \times n$ array whose first row is the permutation $\sigma$ and whose second row is the permutation $\tau$. For $k=1,2, \ldots, n$, connect by a line the occurrence of $k$ in the first row with the occurrence of $k$ in the second row. Then $\{k, l\}$ is an edge of $G_{\sigma, \tau}$ if and only if the line joining the $k$ 's intersects the line joining the l's. If $\sigma=(1,2, \ldots, n)$, then the edges of the graph $G_{\sigma, \tau}$ correspond to the inversions in $\tau$, that is, are the pairs $\{k, l\}$ such that $k<l$ and $j_{k}>j_{l}$. 
Example 3.1. Let $\sigma=(3,1,4,2)$ and $\tau=(2,4,3,1)$. Then $G_{\sigma, \tau}$ is the graph with vertices $1,2,3,4$ and edges $\{1,2\},\{1,4\},\{2,3\},\{2,4\},\{3,4\}$, that is, the complete graph $K_{4}$ minus an edge.

In [17] bipartite graphs which are also permutation graphs are characterized and an algorithm to recognize if a bipartite graph is a permutation graph in linear time is developed. In terms of the biadjacency matrix $A$ of the bipartite graph, both of the following properties characterize those bipartite graphs which are permutation graphs, and it is easy to see that each of these is equivalent to there existing permutation matrices $Q_{1}$ and $Q_{2}$ such that $Q_{1} A Q_{2}$ is a staircase $(0,1)$-matrix.

Let $G(U, W)$ be a bipartite graph with vertex bipartition given by $U, W$. Then the following are equivalent $([17])$ :

(I) $G$ is a permutation graph.

(II) There is an ordering of the vertices of $U$ and those of $W$ such that if

$$
\left[\begin{array}{ll}
* & 1 \\
1 & *
\end{array}\right]
$$

is a $2 \times 2$ submatrix of the biadjacency matrix of $G(U, W)$, then both $*$ 's equal 1.

(III) There is an ordering of the vertices of $U$ and those of $W$ such that (i) for each vertex $x$ in $W$ the vertices joined to $x$ by an edge are consecutive in $U$, and (ii) for every pair $x, y$ of vertices in $W$, if the vertices joined to $x$ are a subset of the vertices joined to $y$, then those vertices joined to $y$ but not to $x$ are consecutive in $U$.

Example 3.2. The following matrix is a staircase (0,1)-matrix illustrating the properties II and III above:

$\left[\begin{array}{l|l|l|l|l|l|l}1 & 1 & 1 & & & & \\ \hline 1 & 1 & 1 & 1 & & & \\ \hline & 1 & 1 & 1 & & & \\ \hline & & 1 & 1 & 1 & & \\ \hline & & 1 & 1 & 1 & 1 & \\ \hline & & 1 & 1 & 1 & 1 & \\ \hline & & & 1 & 1 & 1 & 1\end{array}\right]$.

The matrix

$\left[\begin{array}{l|l|l|l|l} & 1 & 1 & 1 & \\ \hline 1 & 1 & 1 & 1 & 1 \\ \hline 1 & 1 & 1 & 1 & \end{array}\right]$


is convex but does not satisfy II or III.

Properties II and III above characterize bipartite graphs which are permutation graphs. Now consider the matrix

$$
A=\left[\begin{array}{l|l|l|l|l} 
& 1 & 1 & 1 & \\
\hline 1 & 1 & 1 & 1 & 1 \\
\hline 1 & 1 & 1 & 1 &
\end{array}\right] .
$$

Then this matrix is convex, but does not satisfy (II) nor does it satisfy (ii) of (III). To have these properties hold, we need to add two more 1's as in

$$
A^{\prime}=\left[\begin{array}{l|l|l|l|l}
1 & 1 & 1 & 1 & \\
\hline 1 & 1 & 1 & 1 & 1 \\
\hline 1 & 1 & 1 & 1 & 1
\end{array}\right] .
$$

Bipartite graphs which are permutation graphs are characterized above but a permutation graph need not be bipartite. The following theorem gives a simple characterization of permutation graphs which are bipartite.

Theorem 3.3. Let $\sigma$ and $\tau$ be two permutations of $\{1,2, \ldots, n\}$. Then the permutation graph $G_{\sigma, \tau}$ is a bipartite graph if and only if there do not exist distinct integers $a, b, c$ in $\{1,2, \ldots, n\}$ such that $a, b, c$ occur in opposite orders in $\sigma$ and $\tau$.

Proof. If there exist distinct integers $a, b, c$ in $\{1,2, \ldots, n\}$ such that $a, b, c$ occur in opposite orders in $\sigma$ and $\tau$, then the lines joining the $a$ 's, the $b$ 's, and the $c^{\prime}$ 's in the permutation diagram $D_{\sigma, \tau}$ pairwise intersect giving a cycle of length 3 in $G_{\sigma, \tau}$. Thus $G_{\sigma, \tau}$ is not bipartite.

For the converse, we first observe that $G_{\sigma, \tau}$ is isomorphic to $G\left(\iota_{n}, \sigma^{-1} \tau\right)$ where $\iota_{n}$ is the identity permutation of $\{1,2, \ldots, n\}$ : Suppose that $\sigma(i)=p$ and $\sigma(j)=q$ where $i<j$, and $\tau(k)=q$ and $\tau(l)=p$ where $k<l$, so that in $D(\sigma, \tau)$ the lines joining the $p$ 's and $q$ 's intersect. Then $\sigma^{-1}(p)=i$ and $\sigma^{-1}(q)=j$. Also $\sigma^{-1} \tau(k)=\sigma^{-1}(q)=j$ and $\sigma^{-1} \tau(l)=\sigma^{-1}(p)=i$. Thus in $D\left(\iota_{n}, \sigma^{-1} \tau\right)$, the lines joining $i$ and $j$ intersect, and conversely. By this observation we may now assume that $\sigma=\iota_{n}$.

Suppose that there do not exist distinct integers $a, b, c$ in $\{1,2, \ldots, n\}$ such that $a, b, c$ occur in opposite order in $\tau$, that is, $\tau(i)=a, \tau(j)=b, \tau(k)=c$ where $1 \leq i<j<k \leq n$ and $c<b<a$ This means that in the partial order on the set $\{1,2, \ldots, n\}$ where $i<j$ if and only if $\sigma(i)<\sigma(j)$, there does not exist a chain of length 3. By a theorem of Mirsky [8], this means that the elements of $\{1,2, \ldots, n\}$ 
can be partitioned into two antichains (sets of incomparable elements). This gives a bipartition of the vertices of $G\left(\iota_{n}, \tau\right)$ showing that $G\left(\iota_{n}, \tau\right)$ is a bipartite graph.

Recall that a permutation $\tau=\left(j_{1}, j_{2}, \ldots, j_{n}\right)$ of $\{1,2, \ldots, n\}$ is a 321-avoiding permutation provided that there does not exist integers $p<q<r$ such that $j_{p}>$ $j_{q}>j_{r}$, that is, provided that $\tau$ does not contain a decreasing subsequence of length 3.

Corollary 3.4. Let $\sigma=\iota$ be the identity permutation of $\{1,2, \ldots, n\}$ and let $\tau$ be an arbitrary permutation of $\{1,2, \ldots, n\}$. Then $G_{\iota, \tau}$ is a bipartite graph if and only if $\tau$ is a 321-avoiding permutation.

The number of 321-avoiding permutations is the $n$th Catalan number $C_{n}=$ $\frac{1}{n+1}\left(\begin{array}{c}2 n \\ n\end{array}\right)$, see $[1]$.

\section{Sparse ASMs}

In this section we are interested in determining classes of ASMs that have a $(0,1)$ SNF. We first make the following observation.

Let $A$ be an $n \times n(0, \pm 1)$-matrix. We say that $A$ is triangular permutable if there exist permutation matrices $P$ and $Q$ such that $P A Q$ is upper triangular and each row with a zero on the diagonal is a zero row.

Lemma 4.1. Let $A=\left[a_{i j}\right]$ be an $n \times n$ triangular permutable $(0, \pm 1)$-matrix. Then $A$ has a $(0,1)-S N F$.

Proof. Since permuting rows and columns is an EO, we may assume that $A$ is upper triangular and each row with a zero on the main diagonal is a zero row. For $j=1,2, \ldots, n-1$ such that $a_{j j}= \pm 1$, we may add \pm 1 times the $j$ 'th column to each of the next columns, to get zeros in entries $(j, j+1), \ldots,(j, n)$. All rows $j$ for which $a_{j j}=0$ are zero by assumption. Eventually, by this process and after suitable EOs, $A$ is transformed to $I \oplus O$.

A simple triangular elimination algorithm may be used to check if $A$ is triangular permutable: Locate an entry, if any, which is the only nonzero in a row or column, and zero out the nonzero entries in the nonzero row or column just mentioned. Repeat this process on the new matrix. If this process can be carried through to obtain a subpermutation matrix, the original $A$ is triangular permutable, and therefore has a $(0,1)-\mathrm{SNF}$. 
Example 4.2. Applying the triangular elimination algorithm to the ASM below using positions $(6,2),(5,3),(2,5)$ and $(3,4)$ results in:

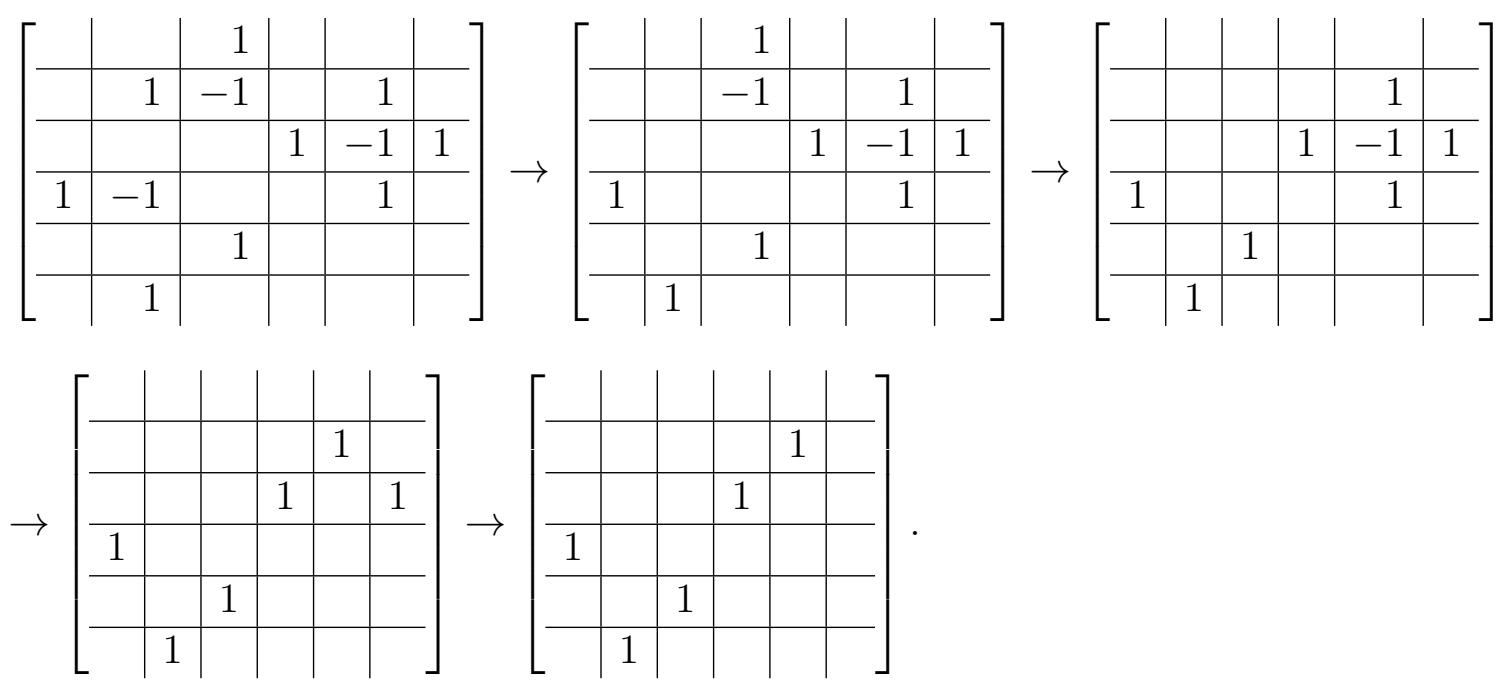

So, $A$ is triangular permutable and $\operatorname{SNF}(A)=I_{5} \oplus O_{1}$.

Let $A$ be an ASM. We say that $A$ is sparse provided it contains at most three nonzeros in every row and column, and thus has at most one -1 in each row and column. The matrix $A$ in Example 1.2 is a sparse ASM, but it does not have a $(0,1)$-SNF. We now investigate sparse ASMs which have a $(0,1)$-SNF.

Consider a sparse ASM $A$. Two nonzero entries in the same row or column of $A$ are called neighbors if the entries between them are all zeros. Every $(-1)$-entry has four neighbors, but a 1-entry has 0,1 or 2 neighbors. A 1-entry with exactly one neighbor will be a called a leaf. A bipartite-cycle in $A$ is a sequence of positions

$$
\left(i_{1}, j_{1}\right),\left(i_{1}, j_{2}\right),\left(i_{2}, j_{2}\right),\left(i_{2}, j_{3}\right), \ldots,\left(i_{1}, j_{t}\right),\left(i_{1}, j_{1}\right)
$$

each containing a 1 in $A$ where, for some $t \geq 2, i_{1}, i_{2}, \ldots, i_{t}$ are distinct row indices and $j_{1}, j_{2}, \ldots, j_{t}$ are distinct column indices. We say that the cycle has length $t$. A cycle matrix is a $(0,1)$-matrix which contains a unique bipartite-cycle.

Theorem 4.3. Let $A$ be a sparse $A S M$ and assume that every (-1)-entry has a neighbor which is a leaf. Then $A$ has a $(0,1)-S N F$ if and only if $A$ does not have a bipartite-cycle of any odd length $t \geq 3$.

Proof. Let $(i, j)$ be an entry (if any) which is -1 , and, say, $(i, k)$ is a neighbor which is a leaf (a similar argument works if the neighbor is in the same column). Since $(i, k)$ is a leaf, column $k$ does not contain a -1 and therefore the 1 in position 
$(i, k)$ is the unique nonzero in column $k$. Now, add column $k$ to column $j$, resulting in a matrix which equals $A$ in every position, except the specified entry $(i, j)$ is 0 . We repeat this process for every negative entry in the matrix, and after these EOs, we have the matrix $B=A^{+}$where all negative entries have been replaced with 0's. Thus, $B$ is a $(0,1)$-matrix with at most two 1 's in every row and column.

If a row in $B$ contains two 1's, where (at least) one is a leaf, we can subtract the column containing the leaf from the other column, and thereby replace a 1 by a 0 in the matrix. A similar operation can be used if a column contains two 1's where at least one is a leaf. After carrying out such EOs, and suitable permutations, the resulting matrix is a direct sum of the following matrices: (i) an identity matrix $I_{s}$ for some $s$, (ii) a zero matrix $O_{p}$ for some $p$, and (iii) cycle matrices. Note that, in this process, no 1 in a bipartite-cycle has been changed into a 0 . Now, every cycle matrix of length 2 can be reduced to the matrix

$$
\left[\begin{array}{ll}
1 & 0 \\
0 & 0
\end{array}\right] .
$$

So after this, we have a matrix which is the direct sum of an identity matrix, a zero matrix, and some cycle matrices (if any) $C_{t}$ corresponding to cycles of length $t \geq 3$. The Smith Normal Form of a cycle matrix $C_{t}$ depends on the parity of $t$. In fact, $\left|\operatorname{det} C_{t}\right|$ is 0 or 2 depending on whether $t$ is even or odd. Moreover, For $1 \leq k<t$, the determinants of the $k \times k$ submatrices of $C_{t}$ are of absolute value 0 or 1 , and for each such $k$ there exists an $k \times k$ submatrix whose determinant has absolute value 1 . Thus if $t$ is even the nonzero determinant divisors $d_{1}, d_{2}, \ldots, d_{t-1}$ of $C_{t}$ are $1,1, \ldots, 1$, while if $t$ is odd the nonzero determinant divisors $d_{1}, d_{2}, \ldots, d_{t}$ of $C_{t}$ are $1, \ldots, 1,2$. The conclusion of the theorem now follows.

Example 4.4. The following sparse ASM $A$ satisfies that every -1 has a neighbor which is a leaf, and clearly has no 1-cycle (of any length).

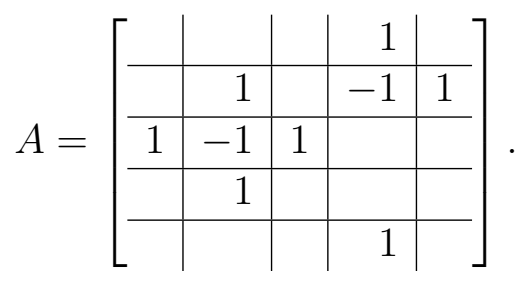

So, by the theorem, $A$ has a $(0,1)-\mathrm{SNF}$.

We now construct a large class of sparse ASMs. To motivate the construction, we first make an observation. Let $A=\left[a_{i j}\right]$ be an ASM and assume that $a_{i j}=-1$ for some position $(i, j)$. Then the nearest nonzero from position $(i, j)$ in row $i$, left 
or right, as well as in column $j$, up or down, must be a 1 . We call the set $S$ of these five positions a star in $A$ with center $(i, j)$ whose boundary consists of the set of four positions of $S$ containing a 1 . Thus, if $A$ has $k$ entries that are -1 , then $A$ contains a configuration of $k$ stars, where the stars may intersect in their boundaries (and such that no 1's are consecutive ignoring 0's). These intersections of the boundaries may be very complex. For the class we now define, these intersections are restricted.

Let $A$ be a sparse ASM with $k$ entries that are -1 (so the -1 's are in a subpermutation pattern) with corresponding stars $S_{1}, S_{2}, \ldots, S_{k}$ and boundaries $B_{1}, B_{2}, \ldots, B_{k}$ such that

(*) $B_{t+1}$ intersects exactly one of the stars $S_{j}$ with $1 \leq j \leq t$, say $S_{i}$, and then $\left|B_{t+1} \cap B_{i}\right| \in\{1,2\}(t<k)$.

Thus, if we construct a graph with vertex set $\left\{S_{1}, S_{2}, \ldots, S_{k}\right\}$ where, for $i \neq j$, a $S_{i}$ and $S_{j}$ form an edge $\left\{S_{i}, S_{j}\right\}$ whenever they intersect, then this graph is a tree, denoted $T_{A}$. We then call such an $A$ a tree-like $A S M$. We label an edge $e=\left\{S_{i}, S_{j}\right\}$ with $l_{e}$, defined as the number of positions common in $S_{i}$ and $S_{j}$; thus $l_{e}=1$ or 2 . For each vertex in $T_{A}$ the sum of the labels of its incident edges is at most 4.

Example 4.5. The following matrix $A$ is a tree-like ASM

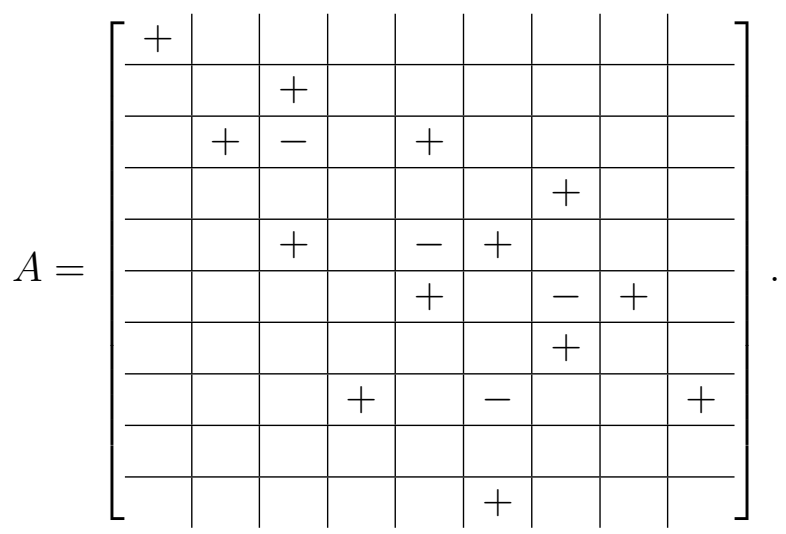

Let $S_{1}, S_{2}, S_{3}, S_{4}$ be the stars with centers $(3,3),(5,5),(6,7)$ and $(8,6)$, respectively. Then $T_{A}$ is the tree with edges $\left\{S_{1}, S_{2}\right\},\left\{S_{2}, S_{3}\right\}$, and $\left\{S_{2}, S_{4}\right\}$, and so it is a (graphtheoretic) star with center $S_{2}$. The labels of these edges, in the given order, are 2 , 1 , and 1 .

Theorem 4.6. We have:

(i) Each tree-like ASM is totally unimodular, and, therefore, it has a $(0,1)-S N F$. 
(ii) Let $T$ be a tree with edge labels 1 and 2 such that, for each vertex, the sum of the labels of its incident edges is at most 4 . Then $T=T_{A}$ for some tree-like $A S M A$.

Proof. First we prove (i). Let $A$ be a tree-like ASM. The proof will be by induction on the number $k$ of stars making up $A$. We use the notation above.

Consider first the case when the last $S_{k}$ that was added, in the construction of $A$, is such that $\left|B_{k} \cap B_{s}\right|=1$ for some $s<k$. Then, after permuting some rows and some columns, $A$ has the following form, or the transpose of this,

$$
A=\left[\begin{array}{l|l}
A_{11} & A_{12} \\
\hline A_{21} & A_{22}
\end{array}\right]=\left[\begin{array}{l|l|l}
+ & \\
- & \\
+ & - & + \\
\hline & + &
\end{array}\right]
$$

where the block $A_{11}$ corresponds to the permuted ASM before the star $S_{k}$ was added, and $A_{22}$ is a $2 \times 2$ matrix. All the unspecified entries in $A_{12}, A_{21}$, and $A_{22}$ equal zero. The final row of $A$ was an earlier row, before the row permutation. Note that any minor of $A_{11}$ is a minor of a (permuted) tree-like ASM with $k-1$ stars. Let $I_{1}$ and $I_{2}$ be the row index sets corresponding to $A_{11}$ and $A_{21}$, respectively. Similarly, let $J_{1}$ and $J_{2}$ be the column index sets corresponding to $A_{11}$ and $A_{12}$, respectively. Consider a $t \times t$ submatrix $C$ of $A$. There are different cases to consider.

(i) If $C$ contains two rows in $I_{2}$, then, as the last two rows of $A$ are equal, $\operatorname{det}(C)=0$.

(ii) If $C$ contains exactly one row in $I_{2}$ and one column in $J_{2}$, then $\operatorname{det}(C)$ is either 0 (when the last column was chosen) or it equals a minor of order $t-1$ in $A_{11}$ (when the first column was chosen). If $C$ contains exactly one row in $I_{2}$ and both columns in $J_{2}$, then

$$
\operatorname{det}(C)=\operatorname{det}(M) \cdot \operatorname{det}\left(\left[\begin{array}{rr}
-1 & 1 \\
1 & 0
\end{array}\right]\right)=-\operatorname{det}(M)
$$

where $M$ is a square submatrix of $A_{11}$ of order $t-2$. Finally, if $C$ contains exactly one row in $I_{2}$, and no column in $I_{2}$, then the last row of $C$ is zero, so $\operatorname{det}(C)=0$.

(iii) If $C$ contains no row in $I_{2}$, but at least one column in $J_{2}$, then, since only the last row in $A_{12}$ is nonzero, it is easy to see that $\operatorname{det}(C)$ is either 0 or \pm some minor of $A_{11}$ of order $t-1$.

(iv) If $C$ has no row in $I_{2}$ and no column in $J_{2}$, then $\operatorname{det}(C)$ is a minor of $A_{11}$. 
Next, consider the case when the last $S_{k}$ that was added, in the construction of $A$, is such that $\left|B_{k} \cap B_{s}\right|=2$ for some $s<k$. Then, after permuting some rows and some columns, $A$ has the form

$$
A=\left[\begin{array}{l|l}
A_{11} & A_{12} \\
\hline A_{21} & A_{22}
\end{array}\right]=\left[\begin{array}{ll|l} 
& \\
++ & - & + \\
+
\end{array}\right] .
$$

All the unspecified entries in $A_{12}, A_{21}$, and $A_{22}$ equal zero. Here the matrix obtained from the leading $(n-1) \times(n-1)$ submatrix of $A$ by replacing the entry in the lower right corner by 0 corresponds to the permuted tree-like ASM with $k-1$ stars. Let $I_{1}, I_{2}, J_{1}$ and $J_{2}$ be defined as above, and let $C$ be a $t \times t$ submatrix of $A$. Again we consider different cases.

(i) Assume $C$ contains both rows in $I_{2}$ and both columns in $J_{2}$. Then we can use EOs based on the last row or column of $C$ to see that $\operatorname{det}(C)$ equals the determinant of a submatrix of order $t-2$ of $A_{11}$ times the determinant of the $2 \times 2$ backward identity matrix. So, $\operatorname{det}(C)$ is the negative of a minor of order $t-2$ of $A_{11}$.

(ii) Assume $C$ contains the first row, but not the second row, in $I_{2}$. Then, possibly after some EOs using the nonzero entry in the last columns, we see $\operatorname{det}(C)$ equals a minor of $A_{11}$ (of order $t-1$ or $t-2$ ). Next, if $C$ contains the second row in $I_{2}$, but not the first, then $\operatorname{det}(C)$ is either zero or equal to a minor of $A_{11}$ of order $t-1$.

(iii) Assume $C$ contains no row in $I_{2}$. Then, if $C$ contains the second column in $J_{2}$, its determinant is zero. Otherwise, $\operatorname{det}(C)$ is a minor of $A_{11}$ or order $t-1$ or $t$, depending on whether $C$ contains a column in $J_{2}$.

Thus we have shown that any minor in a tree-like ASM is either zero or \pm a minor of smaller tree-like ASM. Moreover, the tree-like ASM with one star $(k=1)$, namely the matrix $F_{3}^{2}$, is totally unimodular. Hence statement (i) follows by induction.

Next, we prove (ii) in the theorem by induction on the number of vertices of the tree $T$. If $T$ is a single vertex, then $T=T_{A}$ for $A=F_{3}^{2}$. Now assume that $T$ has at least two vertices. Since $T$ is a tree, it has a leaf $v$ (a vertex of degree 1), and we let $T^{\prime}$ be the tree obtained by removing $v$ and its incident edge $\{v, w\}$. By induction, there is a tree-like ASM $A^{\prime}$ of order $n$ with $T_{A^{\prime}}=T^{\prime}$.

Assume first that $l_{v w}=1$. Then there exists a position $(i, j)$ in the boundary of the star associated with vertex $w$ such that row $i$ (or column $j$, for which a similar argument works) has its only nonzero in position $(i, j)$. Then construct $A=\left[a_{i j}\right]$ 
from $A^{\prime}$ by adding two rows, indexed by 0 and $n+1$, and two columns, indexed by $n+1$ and $n+2$, and letting

$$
a_{i, n+1}=-1, a_{i, n+2}=a_{0, n+1}=a_{n+1, n+1}=1 .
$$

Next, assume that $l_{v w}=2$. Let $S$ be the star associated with vertex $w$ in $T^{\prime}$. Since the sum of the labels for edges incident to $w$ in $T$ is at most 4, by induction, we can choose a tree-like ASM $A^{\prime}$ such that two positions $(i, j)$ and $\left(i^{\prime}, j^{\prime}\right)$ in $S$, not in the same row or column, both have the property of being the unique nonzero in their row or column. (The last property is obtained by a small symmetry argument in the case when $w$ is incident to two edges with label 1 in $T^{\prime}$ ). Assume $i>i^{\prime}$ and $j^{\prime}>j$ (the other cases are similar). Then construct $A=\left[a_{i j}\right]$ from $A^{\prime}$ by adding one row and column, indexed by $n+1$, and letting

$$
a_{i, j^{\prime}}=-1, a_{i, n+1}=a_{n+1, j^{\prime}}=1 .
$$

Then $A$ is a tree-like ASM and $T_{A}=T$, as desired. So, (ii) follows by induction.

We remark that an ASM which is the direct sum of tree-like ASMs also has a $(0,1)$-SNF.

Consider again the matrix $A$ in Example 1.2, which does not have an $(0,1)$-SNF. We note that $A$ is not tree-like and the graph constructed (as above) for the stars with centers in the -1 's contains a cycle. Thus, although we have not found all sparse ASMs with a $(0,1)$-SNF, this suggests that the tree-like ASMs form a rich family of such ASMs.

Remark 4.7. In our computations for determination of the SNF of certain $(0,1)$ matrices and $(0, \pm 1)$-matrices, the EOs we have used have been such that nonzeros are replaced with 0's but no zeros are replaced with nonzeros. Thus we have something for computation of the SNF that is similar to a "perfect elimination scheme" sometimes possible in Gaussian elimination, that is, reducing a matrix to row-echelon or diagonal form without introducing any fill-in, that is without replacing any zeros with nonzeros (see e.g. [10]).

We conclude with a discussion of a special subclass of the sparse ASMs.

Let $\mathcal{A}_{n}^{\text {td }}$ denote the set of all $n \times n$ tridiagonal ASMs. In particular, $\mathcal{A}_{n}^{\text {td }}$ includes the tridiagonal permutation matrices, studied in [5] along with the tridiagonal doubly stochastic matrices. An $n \times n$ tridiagonal permutation matrix is a special involution; as a permutation of $\{1,2, \ldots, n\}$, it consists of fixed points and transpositions of the form $(i, i+1)$ for some $i$ with $1 \leq i \leq n-1$. Thus a tridiagonal permutation matrix is a direct sum of $1 \times 1$ identity matrices $I_{1}$ and matrices

$$
L_{2}=\left[\begin{array}{ll}
0 & 1 \\
1 & 0
\end{array}\right] \text {. }
$$


The matrices $F_{n}^{2}$ belong to $\mathcal{A}_{n}^{\text {td }}$ where $F_{2}^{2}=L_{2}$ and $F_{1}^{2}=I_{1}$. In fact, it is straightforward to check that $\mathcal{A}_{n}^{\text {td }}$ consists of those ASMs that are direct sums of matrices equal to $I_{1}, L_{2}$, and $F_{m}^{2}$ for $m \geq 3$. In particular, we see that all matrices in $\mathcal{A}_{n}^{\text {td }}$ are symmetric and, by Corollary 2.2 , have a $(0,1)-\mathrm{SNF}$.

Example 4.8. The following $7 \times 7$ matrix is a tridiagonal ASM:

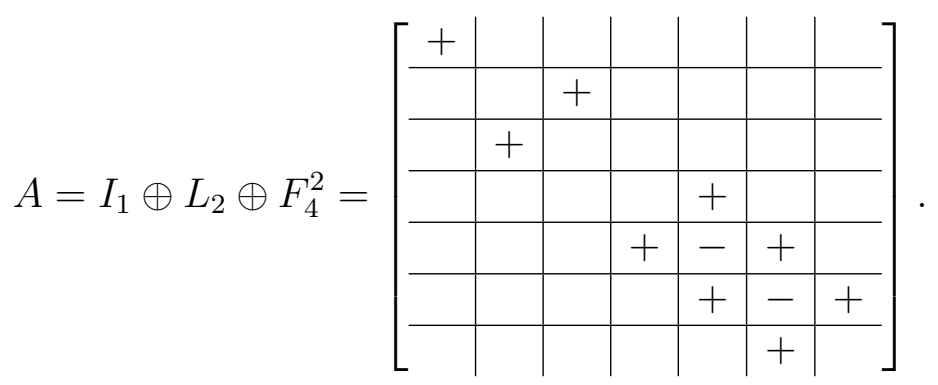

The number of $n \times n$ tridiagonal ASMs is easily counted.

Lemma 4.9. $\left|\mathcal{A}_{n}^{\mathrm{td}}\right|=2^{n-1}$.

Proof. According to our description of the tridiagonal ASMs as direct sums of matrices $F_{m}^{2}$ for $m \geq 1$, an $n \times n$ tridiagonal ASM is determined by a representation of $n$ as a sum of one or more positive integers:

$$
n=n_{1}+n_{2}+\cdots+n_{k} \leftrightarrow F_{n_{1}}^{2} \oplus F_{n_{2}}^{2} \oplus \cdots \oplus F_{n_{k}}^{2} .
$$

The number of such representations (5) equals $\left(\begin{array}{l}n-1 \\ k-1\end{array}\right)$ (take a sequence of $n$ 1's and choose $(k-1)$ out of the $(n-1)$ distinct places between them to determine the $n_{i}$ 's) and thus

$$
\left|\mathcal{A}_{n}^{\mathrm{td}}\right|=\sum_{k=1}^{n}\left(\begin{array}{l}
n-1 \\
k-1
\end{array}\right)=2^{n-1} .
$$

In fact, there is a bijection between the set $\mathcal{A}_{n}^{\text {td }}$ and the set of $(0,1)$-vectors of length $n-1$, corresponding to the positions $(i, i+1)$ for $i \leq n-1$.

We also consider a polytope conv $\left(\mathcal{A}_{n}^{t d}\right)$, the convex hull of all the $n \times n$ tridiagonal ASMs, and we call this the tridiagonal ASM polytope. It follows from the proof of the above lemma that this polytope is affinely equivalent to the unit cube $[0,1]^{n-1}$. As a consequence of the discussion above, we get a linear characterization of the tridiagonal ASM polytope $\operatorname{conv}\left(\mathcal{A}_{n}^{t d}\right)$. 
Theorem 4.10. $\operatorname{conv}\left(\mathcal{A}_{n}^{t d}\right)$ equals the set of $n \times n$ real matrices $A=\left[a_{i j}\right]$ that are tridiagonal, symmetric and satisfy

$$
\begin{array}{lll}
\text { (i) } \quad 0 \leq a_{i, i+1} \leq 1 & (1 \leq i<n) \\
\text { (ii) } & a_{i i}=1-a_{i, i-1}-a_{i, i+1} & (1 \leq i \leq n)
\end{array}
$$

where we define $a_{10}=a_{n, n+1}=0$.

Acknowledgment. The authors would like to thank a referee for several very useful comments.

\section{References}

[1] M. Bona, Combinatorics of Permutations, Chapman \& Hall/CRC, Boca Raton, 2004.

[2] R.A. Brualdi and G. Dahl, The Bruhat shadow of a permutation matrix, Mathematical Papers in Honour of Eduardo Marques de Sá, O. Azenhas, A.L. Duarte, J.F. Queiró, A.P. Santana, eds., Textos de Mathemática Vol. 39, Dep. de Matemática da Univ. de Coimbra, Portugal 2006, 25-38.

[3] H.-C. Chen and T.Y. Ho, The rook problem on saw-toothed chessboards, Appl. Math. Lett., 21 (2008), 1234-1237.

[4] D. Chistikov, S. Kiefer, I. Marušić, M. Shirmohammadi, and J. Worrell, Nonnegative matrix factorization requires irrationality, SIAM J. Appl. Algebra Geometry, 1 (2017), 285-307.

[5] G. Dahl, Tridiagonal doubly stochastic matrices, Linear Algebra Appl., 390 (2004), 197-208.

[6] M. Fiedler, F.J. Hall, and M. Stroev, Dense alternating sign matrices and extensions, Linear Algebra Appl., 444 (2014) 219-226.

[7] M. Fiedler, W. Gao, F.J. Hall, G. Jing, Z. Li, and M. Stroev, Ranks of dense alternating sign matrices and their sign patterns, Linear Algebra Appl., 471 (2015) 109-121.

[8] L. Mirsky, A dual of Dilworth's theorem, Amer. Math. Monthly, 78 (1971), 876-877. 
[9] M. Fiedler and V. Ptak, Cyclic products and an inequality for determinants, Czechoslovak. Math J., 19 (1969), 428-451.

[10] M.C. Golumbic, Algorithmic Graph Theory and Perfect Graphs, AcademicPress, London, 1980.

[11] T. Jiang and B. Ravikumar, Minimal NFA problems are hard, SIAM J. Comput., 22 (1993), 1117-1141.

[12] R. Karp, Reducibility among combinatorial problems, in Complexity of Computer Computations, Plenum, New York, 1972, 85-103.

[13] B.D. Saunders and H. Schneider, Flows on graphs applied to diagonal similarity and diagonal equivalence for matrices, Disc. Math., 24 (1978), 205-220.

[14] A. Schrijver, Theory of Linear and Integer Programming, Wiley, Chichester, 1986.

[15] Y. Shitov, On the complexity of Boolean matrix ranks, Linear Algebra Appl., 439 (2013), 2500-2502.

[16] Y. Shitov, The nonnegative rank of a matrix: Hard problems, easy solutions, SIAM Review, 59 (2017), 794-800.

[17] J. Spinrad, A. Brandstädt, L. Stewart, Bipartite permutation graphs, Disc. Appl. Math., 18 (3) (1987), 279-292.

[18] R.P. Stanley, Smith normal form in combinatorics, J. Combin. Theory Ser. A, 144 (2016), 476-495.

[19] T. Watson, Nonnegative rank vs. binary rank, arXiv:1603.07779v1 (2016). 